\title{
Hypertrophic Cardiomyopathy
}

\author{
N. B. KARATZAS ${ }^{\star}$, J. HAMILL, AND P. SLEIGHT \\ From the Cardiac Department, Radcliffe Infirmary, Oxford
}

Many different names have been assigned to the myocardial disease which is characterized by ventricular hypertrophy and abnormal function, and which is often associated with obstruction of the outflow of the left ventricle (McMichael, 1964). Goodwin et al. (1960) have proposed the term "obstructive cardiomyopathy" in order to emphasize the generalized nature of the heart muscle disorder, and later the word "hypertrophic" has been added to this description (Cohen et al., 1964) to point out that in some cases obstruction may not be present despite massive ventricular hypertrophy. Whether these latter cases will go on to develop outflow obstruction is not known.

These cases often present diagnostic difficulties, in contrast to the patients with outflow obstruction in whom the diagnosis can usually be made on clinical grounds alone. There are numerous reviews of the obstructive cases, which, as was remarked by Burchell (1964), give almost a monotonous clinical and haemodynamic description of the condition. There has, however, been little emphasis on marginal cases which show little or no obstruction.

We describe 4 such patients who presented diagnostic problems. The clinical presentation in 3 of them did not suggest the diagnosis of hypertrophic obstructive cardiomyopathy, but subsequent investigations indicated that they might belong to the same group. In the fourth, the diagnosis of hypertrophic obstructive cardiomyopathy was suspected on the grounds of family history and clinical signs, but could not be confirmed by further tests.

\section{CASE REPORTS}

Case 1. A housewife aged 24, the twin sister of Case 2. Genetic studies (courtesy of Dr. Ian Shine of the Medical Research Council Population Genetics Research Unit, Oxford) showed that the sisters were probably $(p=0.98)$ monozygotic twins, judged by physical

Received February 24, 1968.

* Present address: Cardiovascular Research Institute, University of California, San Francisco Medical Centre, U.S.A. appearance, 9 blood group systems, finger ridge counts, and palm fold angle (atd angle). Their mother died from tuberculosis when they were young, and the father is apparently healthy. There are no other sibs.

Cardiac enlargement was first noted on routine $x$-ray examination. She had no symptoms, though she thought that she was slightly more short of breath on exertion than her companions; she has never fainted. At the age of 19 she had an uneventful pregnancy.

On examination she was a small woman, and there was a prominent ' $a$ ' wave in the jugular venous pulse. The blood pressure was $120 / 70 \mathrm{~mm}$. $\mathrm{Hg}$, and the arterial pulse had a rapid upstroke. External carotid pulse recordings showed a normal configuration. There was a right ventricular impulse. A short ejection systolic murmur was present. The second heart sound was widely split and there was only minimal variation with respiration. The maximum increase in the splitting of the second heart sound, measured over 60 consecutive cycles at a paper speed of $100 \mathrm{~mm}$./sec. during deep respiration, was $13 \mathrm{msec}$. (Fig. 1). A soft third heart sound was audible at the mitral area. Radiography of the chest showed cardiac enlargement (Fig. 2, Case 1); the left atrium was enlarged, as was the main pulmonary artery. The electrocardiogram showed sinus rhythm, with a prolonged $P-R$ interval $(0.22 \mathrm{sec}$.), large $P$ waves, incomplete right bundle-branch block, and inverted $T$ waves in the praecordial leads (Fig. 3, Case 1).

Cardiac catheterization when she was 22 revealed pulmonary hypertension and high end-diastolic pressures in both ventricles, with large transmitted ' $a$ ' waves (Fig. 4). The pressures in $\mathrm{mm}$. $\mathrm{Hg}$ before angiography were as follows: right atrium mean 10, a 17, × 2, v 12, and $y 5$; right ventricle, $45 / 15$; pulmonary artery $45 / 20$ (mean 30); pulmonary arterial wedge mean 25, a 31, x 22, v 30, and y 19; left ventricle, $134 / 20$ and aorta $134 / 70$ (mean 92). Pulmonary arterial resistance was 1.4 units.

The pattern of the aortic pressure pulse was normal. There were no systolic pressure gradients across the outflow tract, or between cavity and outflow, in either the right or left ventricle, and no diastolic gradient across the mitral valve. The ventricular pressure pulse did not display the notch in the upstroke sometimes seen in obstructive cardiomyopathy. The pause following ectopic beats which occurred during catheterization did not result in a rise in the left ventricular systolic pressure 


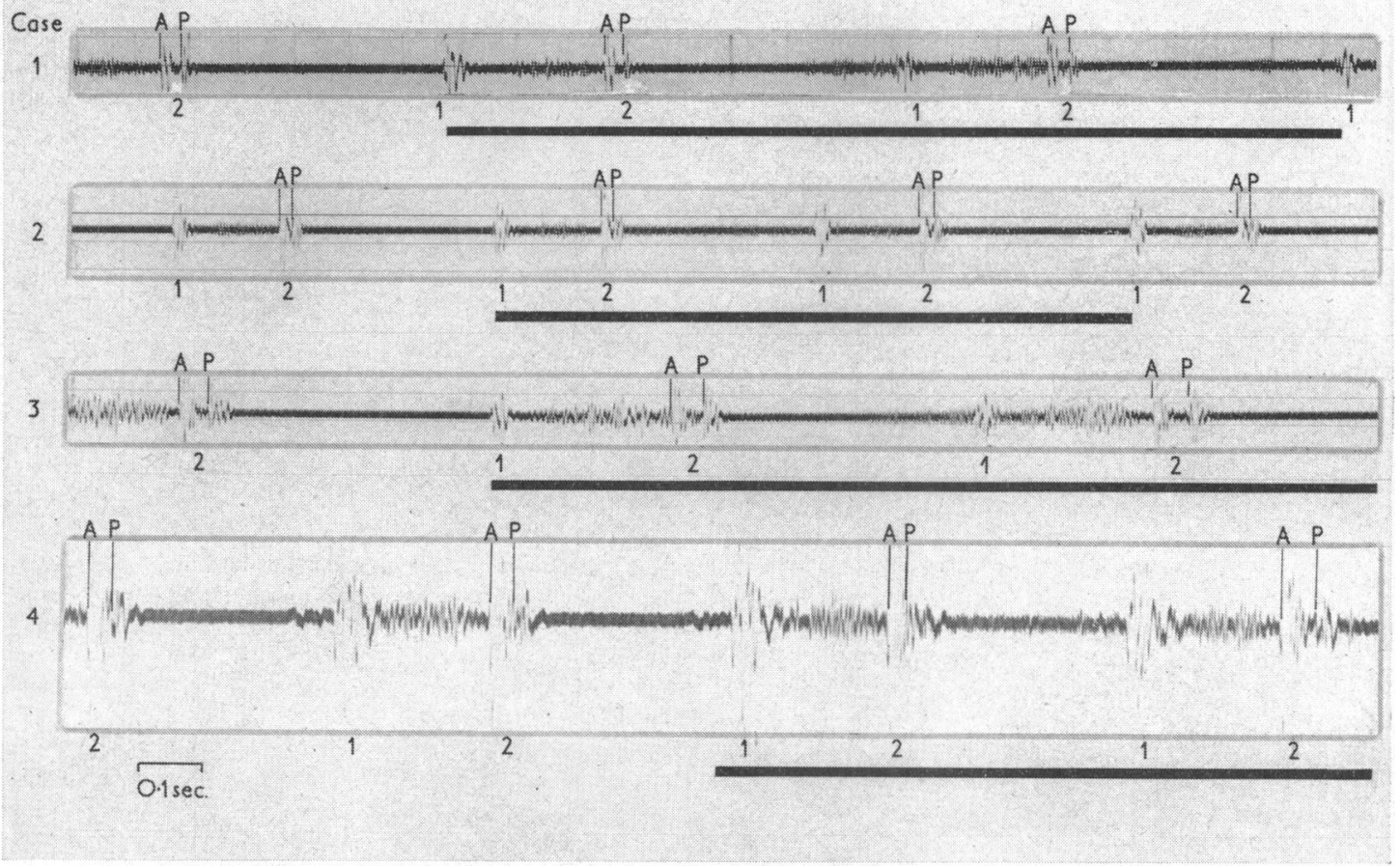

FIG. 1.-The phonocardiogram recorded from the left sternal border in each of the 4 patients described in the text. The thick black lines mark the phase of inspiration. 1 and 2 , the first and second heart sounds, respectively. A and $\mathrm{P}$, the aortic and pulmonary components of the second heart sound.

There were no exercise or other provocative manoeuvres. Left ventricular angiography showed the left ventricular wall to be of normal thickness, except on the inferior and median aspect where there was local thickening and nodularity best seen in early systole (Fig. 5, Case 1), but not obliterated in diastole. There was no evidence of narrowing of the outflow tract at any phase of the cardiac cycle. No shunts were detected during catheterization. The patient has remained symptom free over the $2 \frac{1}{2}$-year period of follow-up.

Comment. The right ventricular impulse, the ejection systolic murmur, and the fixed splitting of the second heart sound, together with the incomplete right bundlebranch block on the electrocardiogram, suggested the diagnosis of atrial septal defect on her first visit. However, the chest radiography was not consistent with this diagnosis. The haemodynamic findings were also inconclusive. The raised right and left ventricular enddiastolic pressures associated with pulmonary hypertension were unexplained. The only evidence of an anatomical lesion was the angiographic appearance of irregular thickening of part of the left ventricular wall.

Case 2. The twin sister of Case 1 is also a housewife. She was more short of breath on exertion than her sister and had attacks of palpitation which started and ended suddenly, lasted one or two minutes, and occurred without obvious precipitating factors. However, like her sister, she never had angina or loss of consciousness. At the age of 19 she too had a normal pregnancy during which there were no symptoms, but the jugular venous pressure was high.

On examination she resembled her sister but there was a raised jugular venous pressure with a large ' $a$ ' wave. The arterial pulse had a rapid upstroke. There was a right ventricular heave and a normal apex beat. A short ejection systolic murmur was just audible, and the second heart sound was widely split, and fixed on respiration (maximal inspiratory increase of $9 \mathrm{msec}$.) (Fig. 1, Case 2). A third heart sound was present at the apex.

The chest radiography showed cardiac enlargement, the left atrium was enlarged, and the vessels of the upper lobes were also slightly enlarged (Fig. 2, Case 2). The electrocardiogram was almost identical with her sister's (Fig. 3, Case 2) and showed large $\mathbf{P}$ waves, long $\mathbf{P}-\mathbf{R}$ interval $(0.22 \mathrm{sec}$.), incomplete right bundle-branch block, and widespread $T$ wave inversion.

Cardiac catheterization, first performed when she was aged 20, showed findings that were similar to those of her sister. The pressures in $\mathrm{mm}$. $\mathrm{Hg}$ were as follows: right atrium mean 12, a 22 , x 8, v 17 , y 9 ; right ventricle $80 / 25$; pulmonary artery $80 / 42$ (mean 68); pulmonary arterial wedge mean 30 , a 35, x $30, \mathrm{v} 42$, y 32 ; femoral artery 
143/81 (mean 121). The femoral artery pressure pulse configuration was normal. The pulmonary arterial resistance was 7 units. No shunts were detected. Right ventricular angiography showed no abnormality. Catheterization was repeated six months later by the transseptal route to investigate the left heart. The pressures in $\mathrm{mm} . \mathrm{Hg}$ were as follows: right atrium mean 14, a 27, x 7, v 19, and y 10; right ventricle $80 / 32$; pulmonary artery 80/44 (mean 60 ); pulmonary arterial wedge mean 35 , a 38 , x 35, v 45, and y 28 ; left atrium mean 34 , a 35 , x 31, v 42, and y 23; left ventricle $120 / 36$; aorta $125 / 73$ (mean 87). The pulmonary arterial resistance was 5.8 units. Left ventricular angiography (Fig. 5, Case 2) showed the left ventricular wall to be abnormally thin laterally, but thick and nodular inferio-medially; there was no narrowing of the outflow tract. A degree of mitral incompetence was shown, but this may have been due to the presence of the catheter through the valve. Exercise or other manoeuvres were not performed during catheterization to provoke obstruction and hence a pressure gradient.

At the age of 20 she had a spontaneous abortion in the fifth month of pregnancy, followed by dilatation and curettage. Seven days later she developed an episode suggestive of acute right ventricular failure, with pain in the right hypochondrium, tenderness and enlargement of the liver, and further rise in jugular venous pressure. Pulmonary embolism was considered, but there was no firm evidence of this. The episode subsided within one week and has not recurred. She has remained slightly breathless on exertion but otherwise symptom free over the follow-up period of 2 years.

Comment. Though this patient appears to be more severely affected than her sister, both seem to be suffering from the same condition.

Case 3. A 13-year-old schoolgirl. During an attack of tonsillitis at the age of 9 a cardiac murmur was noted. 


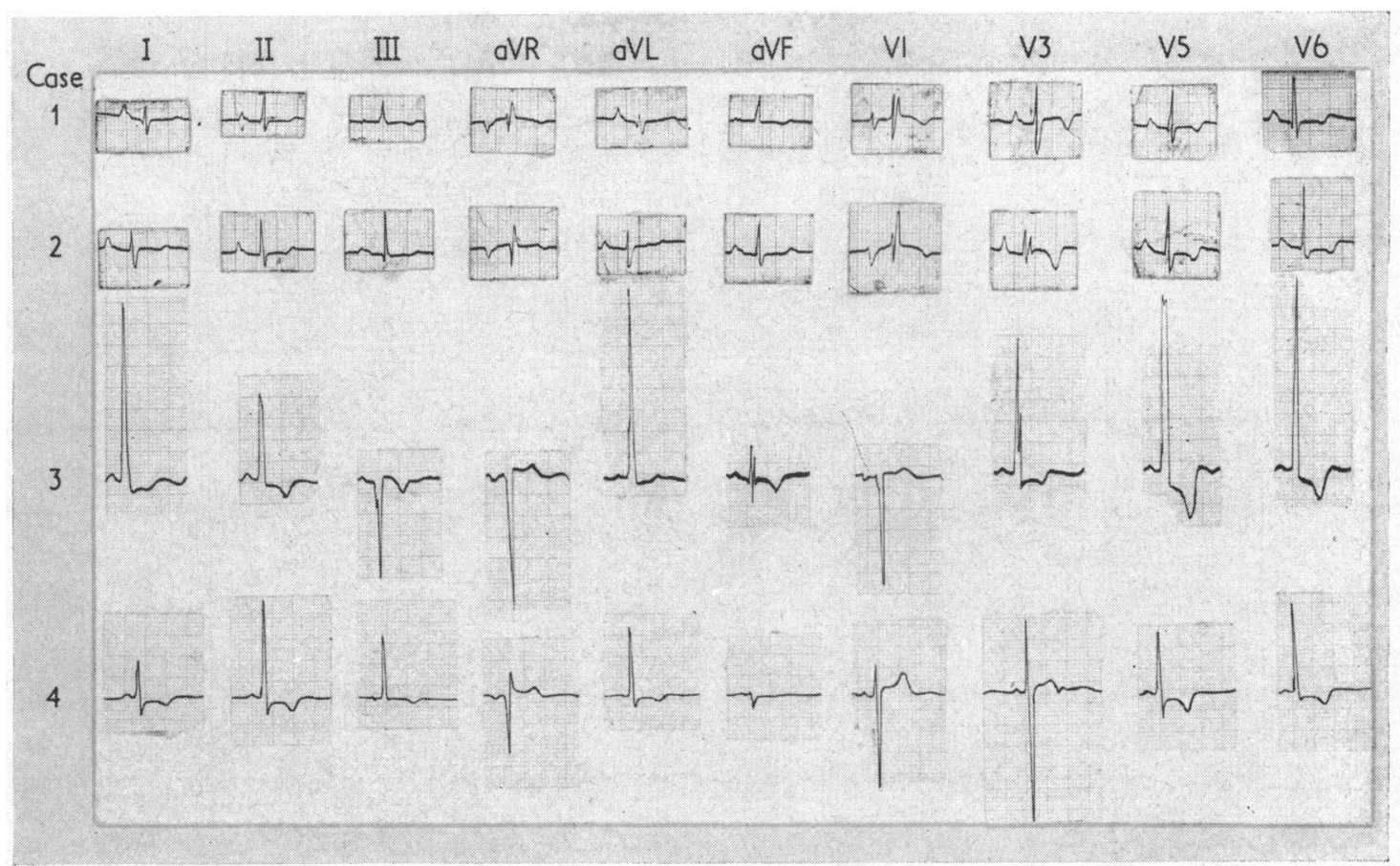

FIG. 3.-The electrocardiograms of the 4 patients. Cases 1 and 2 show delayed AV conduction, incomplete right bundle-branch block, and inverted $T$ waves in the praecordial leads. Case 3 shows left ventricular hypertrophy, and Case 4 widespread S-T depression and T wave inversion.

She was symptom free and never had undue dyspnoea, palpitation, or pains in the chest, and had never fainted. She had 4 sibs; one died of pertussis at the age of 1 year; the others, her two sisters aged 3 and 9 , are normal and have been examined by us. The parents are apparently normal.

On physical examination she was well; the jugular venous pulse, arterial pulse, and external carotid recordings were normal. There was a left parasternal heave. A short ejection systolic murmur and fixed splitting of the second sound were present (maximal inspiratory increase of splitting $8 \mathrm{msec}$.). Radiography of the chest was normal except for a prominence of the left cardiac border (Fig. 2, Case 3). The electrocardiogram showed conspicious left ventricular hypertrophy (Fig. 3, Case 3). Cardiac catheterization when she was 9 showed nothing abnormal. Pressures in $\mathrm{mm}$. $\mathrm{Hg}$ were as follows: right atrium mean 1 , a 3 , x 1, v 3 , y 0 ; right ventricle $23 / 0$; pulmonary artery $23 / 12$ (mean 18); pulmonary arterial wedge mean 13 , a 16, x 14, v 16, and y 11 ; femoral artery 116/75 (mean 88). The pulmonary arterial resistance was 1.5 units. The left ventricle was not catheterized. Angiography (Fig. 5, Case 3) was performed by a right ventricular injection of contrast medium. The right ventricle and the pulmonary vessels were normal. The left ventricular wall was grossly thickened, particularly medially and inferiorly, and the cavity was curved, long, and narrow, but there was no narrowing of the outflow tract.

Several times in subsequent examinations evidence of obstruction was sought during exercise, or during the Valsalva manoeuvre, by recording the intensity of the murmur, the configuration of the arterial pulse, and the blood pressure, but none was obtained.

The patient has remained symptom free for the followup period of four years.

Comment. This patient like the previous ones, had physical signs that suggested the diagnosis of atrial septal defect. The electrocardiogram and the chest radiography, however, were not consistent with this diagnosis. The gross left ventricular hypertrophy seen on the electrocardiogram was confirmed by angiography, but its aetiology remained obscure. Since this girl is symptom free, and there is neither clinical nor radiological suggestion of left ventricular outflow obstruction, it was thought unjustifiable to perform a second cardiac catheterization in order to investigate the possibility of a pressure gradient across the left ventricular outflow tract.

Case 4. A symptom-free and physically active boy, aged 18, was referred because of a family history of early sudden death in his maternal relatives. His mother's 

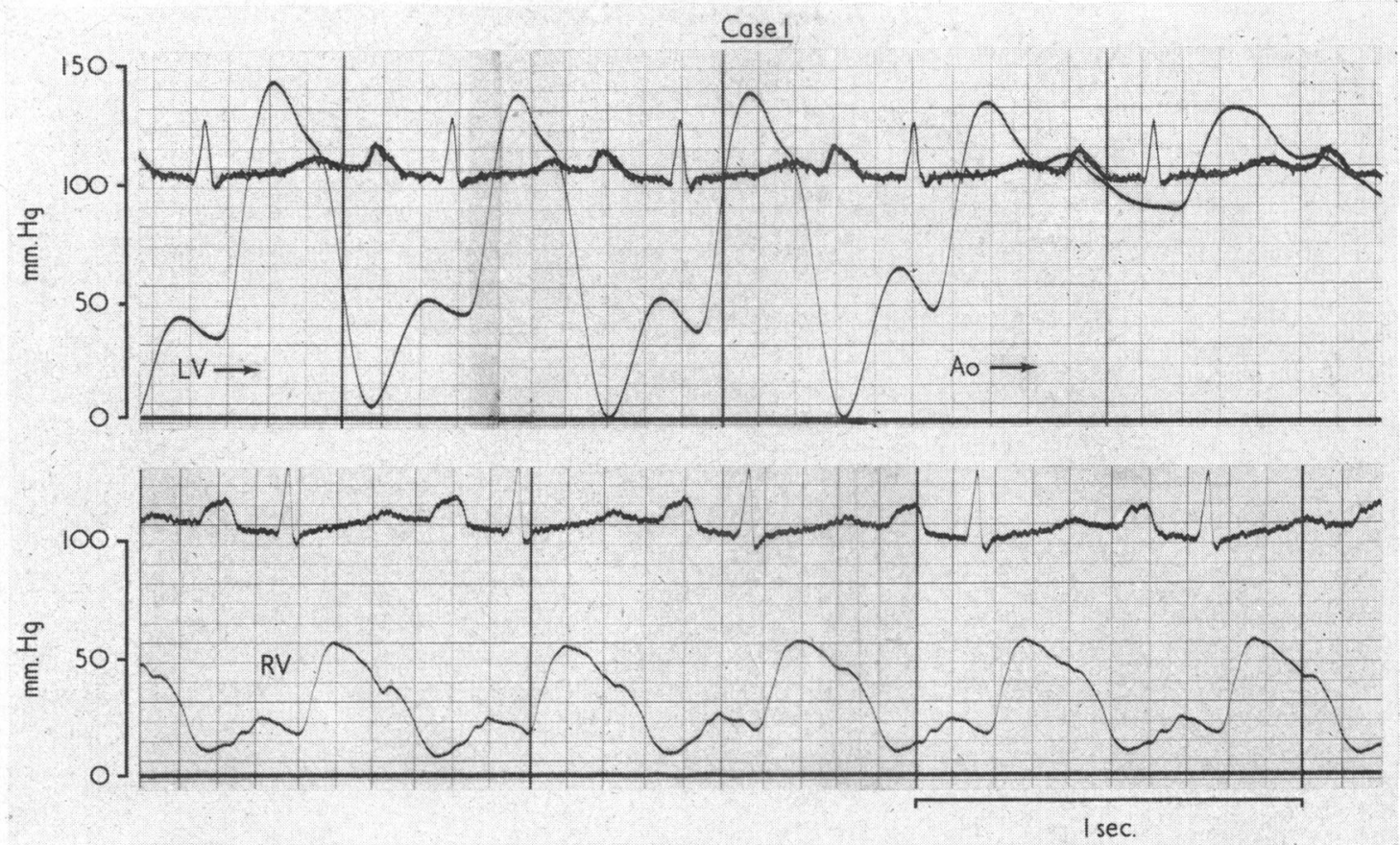

FIG. 4.-Pressure tracings from Case 1. The upper panel shows a withdrawal tracing from the left ventricle to the aorta, and the lower panel a pressure tracing from the right ventricle. There is no systolic pressure gradient between left ventricle and aorta. The end-diastolic pressure is raised in both left and right ventricles, and there are large transmitted ' $a$ ' waves. (The left ventricular and aortic pressure tracings are those obtained after left ventricular angiography.) Similar tracings were obtained from Case 2.

twin brother died suddenly at the age of 39, and necropsy showed considerable left ventricular hypertrophy, with extensive myocardial fibrosis. This uncle had been examined six years earlier because of fainting attacks and no abnormality was found. Both his sons also died suddenly, one at the age of 9 while playing, and at necropsy the heart was enlarged and hypertrophied, but no diagnosis was established. The other son had been perfectly fit and was in the navy when he died suddenly at the age of 20 . Necropsy showed the left ventricle to be hypertrophied, but there was no other abnormality. Another nephew of the patient's mother died suddenly on a football field while in the army at the age of 18 . The mother herself is well, but her electrocardiogram shows incomplete left bundle-branch block.

On physical examination he was well and there were no abnormal findings other than a short ejection systolic murmur at the left sternal edge and a split second heart sound whose components moved normally with respiration (Fig. 1, Case 4). The chest $x$-ray (Fig. 2 Case 4) was normal, though the left cardiac border showed an unusual curvature. The electrocardiogram showed persistent widespread S-T segment depression and $T$ wave inversion (Fig. 3, Case 4).

Cardiac catheterization showed no abnormality apart from a raised end-diastolic pressure in the left ventricle associated with a large transmitted ' $a$ ' wave. The pres- sures in $\mathrm{mm}$. $\mathrm{Hg}$ were as follows: right atrium mean 4.5 , a 6 , x 2 , v $4 \cdot 5$, and y $4 \cdot 5$; right ventricle $26 / 5$; pulmonary artery 26/11 (mean 18); pulmonary arterial wedge mean 12 , a 14, x 6, v 13, and y 8 ; left ventricle $123 / 20$; aorta $123 / 90$ (mean 105); femoral artery 150/77 (mean 105). Pulmonary arterial resistance was 1.0 unit. The femoral arterial and left ventricular pressure responses to the Valsalva maneouvre and to the administration of amyl nitrate and isoprenaline were normal, and no significant systolic pressure gradient was produced by these means (Fig. 6).

Left ventricular cine-angiography showed a normal outflow tract during all phases of the cardiac cycle. The ventricular cavity was normal during diastole, but it contracted to a narrow tapered structure during systole (Fig. 7). The ventricular wall appeared thickened at its supero-lateral aspect. Supravalvular cine-aortography showed normal coronary arteries.

The patient remained symptom free for six months after he was first seen.

Comment. There is little doubt that this boy's relatives who died suddenly suffered from hypertrophic cardiomyopathy. The boy himself appears to be mildly affected by the condition, which explains the electrocardiographic and radiographic findings and the rise in left ventricular end-diastolic pressure. 


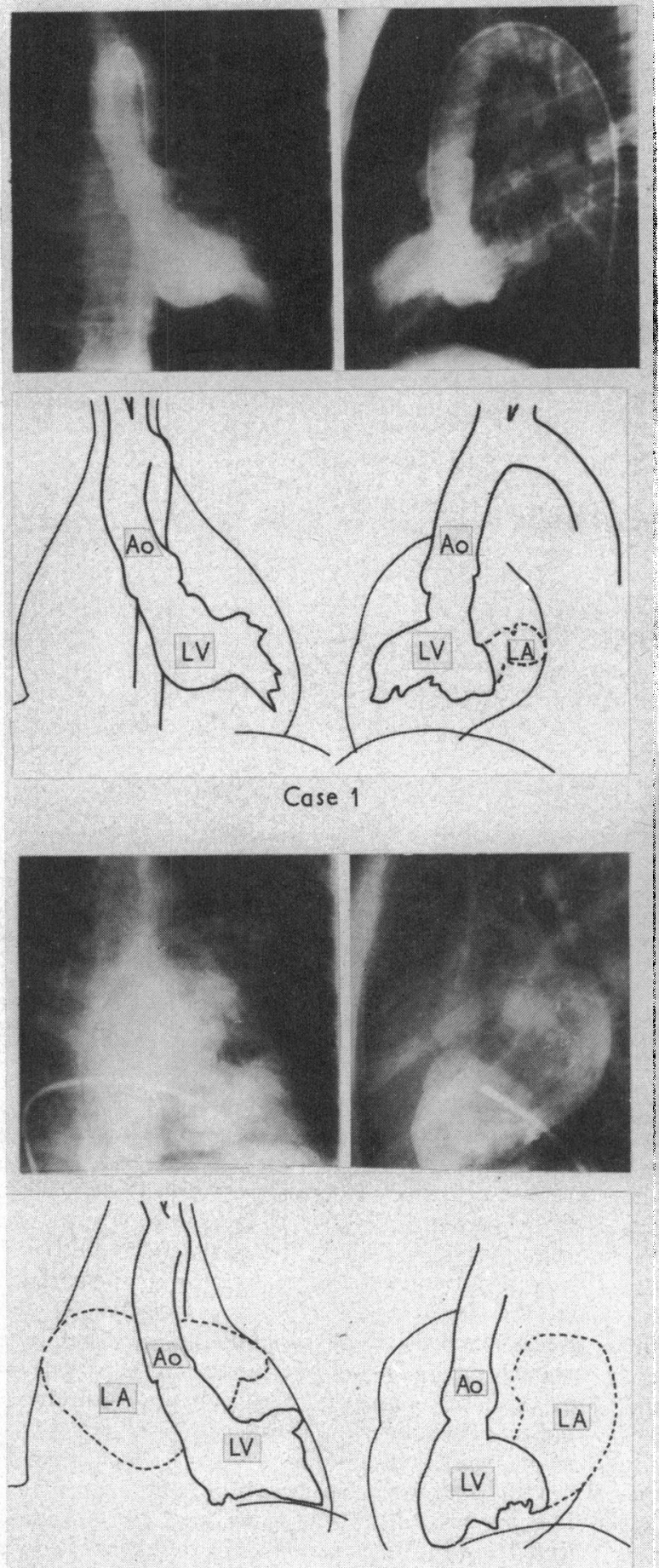

Case 2
Fig. 5.1-Frontal and lateral views of the left ventricular angiogram of Case 1 taken during systole, with outline tracings. There is no outflow tract narrowing. The floor of the left ventricle is irregular, thickened, and encroaches on the cavity.

Fig. 5.2-Frontal and lateral views of a left ventricular angiogram obtained during the systolic phase from Case 2, with outline tracings. The position of the transseptal catheter is seen. Contrast medium opacifies the left atrium. There is no outflow tract narrowing but the floor of the left ventricle is nodular and hypertrophied.

Fig. 5.3.-Frontal and lateral views of the left ventricular phase of a right ventricular angiogram from Case 3, with outline tracings. There is no outflow tract narrowing but the left ventricular cavity appears elongated and narrowed.
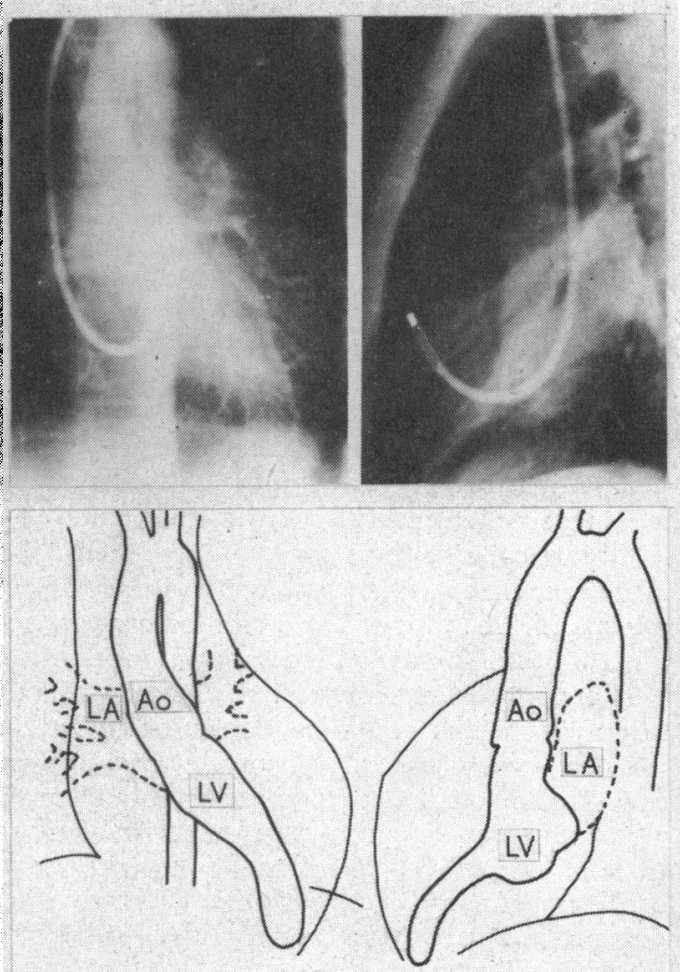

Cose 3 


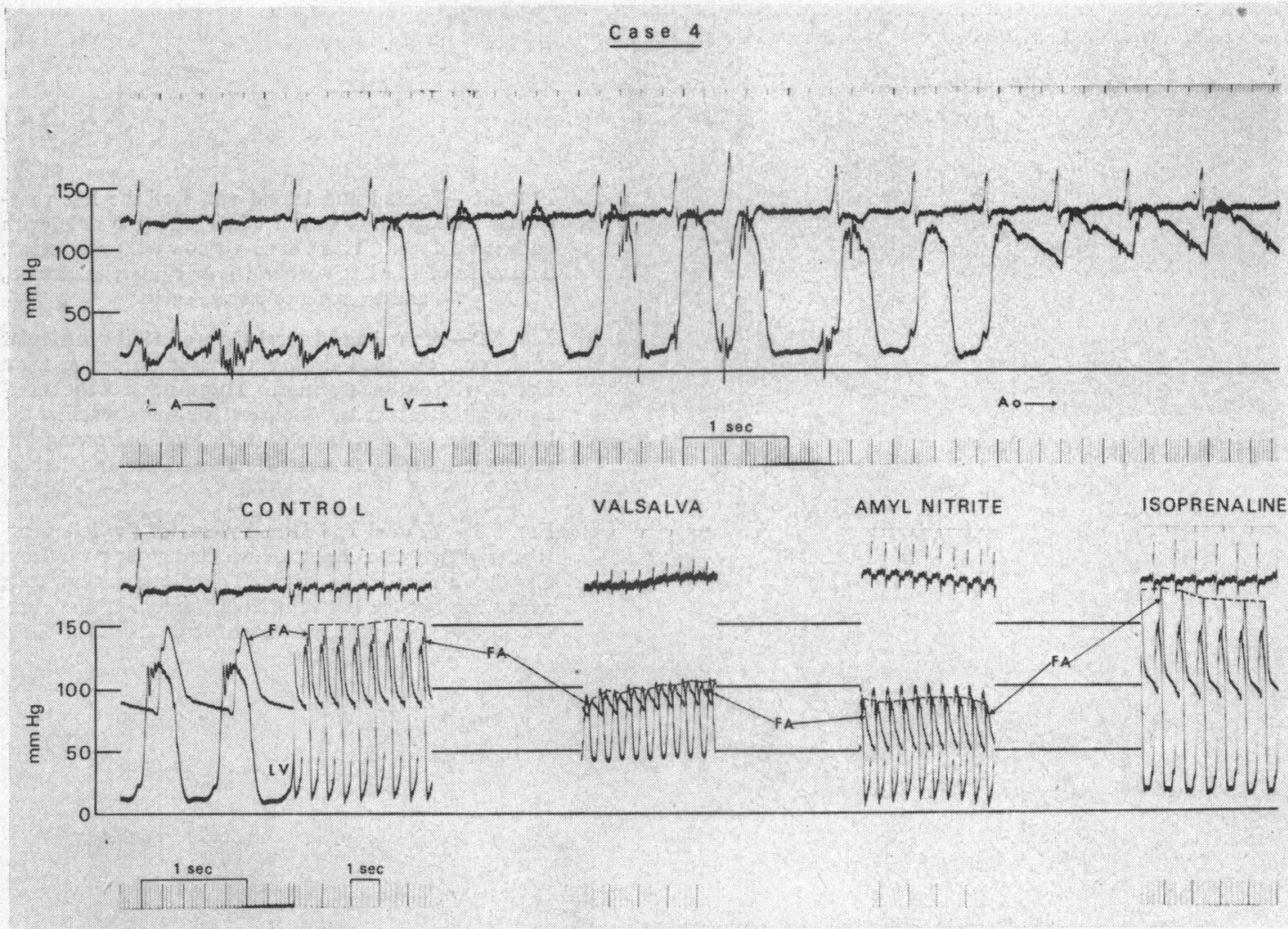

Fig. 6.-Pressure tracing from Case 4. The upper panel shows a withdrawal tracing from the left atrium to the aorta 15 minutes after isoprenaline administration. The catheter entered the left atrium through the mitral valve. There is no intracavitary or outflow tract gradient.

The lower panel shows left ventricular and femoral arterial pressures at rest, during the Valsalva manoeuvre, during the period of maximal pressure drop after amyl nitrate inhalation, and 10 minutes after administration of $40 \mathrm{mg}$. isoprenaline, sublingually. The dashed line marks the peak of the femoral arterial pressure pulse.

\section{Discussion}

The diagnosis of hypertrophic obstructive cardiomyopathy is usually made on the basis of symptoms and signs which result from the obstruction of the left ventricular outflow tract. These signs, if absent at the time of examination, may be provoked by various means. Since the condition appears to be inherited, the family history is also important.

None of the 4 patients we have described had any symptoms which could be attributed to left ventricular outflow tract obstruction, nor was obstruction found at cardiac catheterization, though provocative manoeuvres were performed in only one patient. However, all 4 patients had electrocardiographic and angiographic evidence of left ventricular hypertrophy, and 3 of the 4 had a strong family history of this disease.

Cardiac catheterization provided no explanation for the cardiac hypertrophy, but the left ventricular end-diastolic pressure was raised in 3 of the 4 patients despite the absence of clinical evidence of cardiac failure. There was no clinical or angiographic evidence of coronary artery disease in any of the patients, though selective coronary arteriography was not done. The cardiac hypertrophy in these 4 patients, therefore, appeared unexplained, but the angiographic appearances of the left ventricular cavity closely resembled those that often accompany the left ventricular outflow tract narrowing seen on angiography in patients with hypertrophic obstructive cardiomyopathy.

The occasional absence of outflow tract obstruction in this condition has been noted previously (Braunwald et al., 1964; Cohen et al., 1964). Indeed, Nasser et al. (1967) examined 33 members of a large family and found 15 of them to be affected by hypertrophic cardiomyopathy. In 7 of these 15 members, however, systolic pressure gradients 


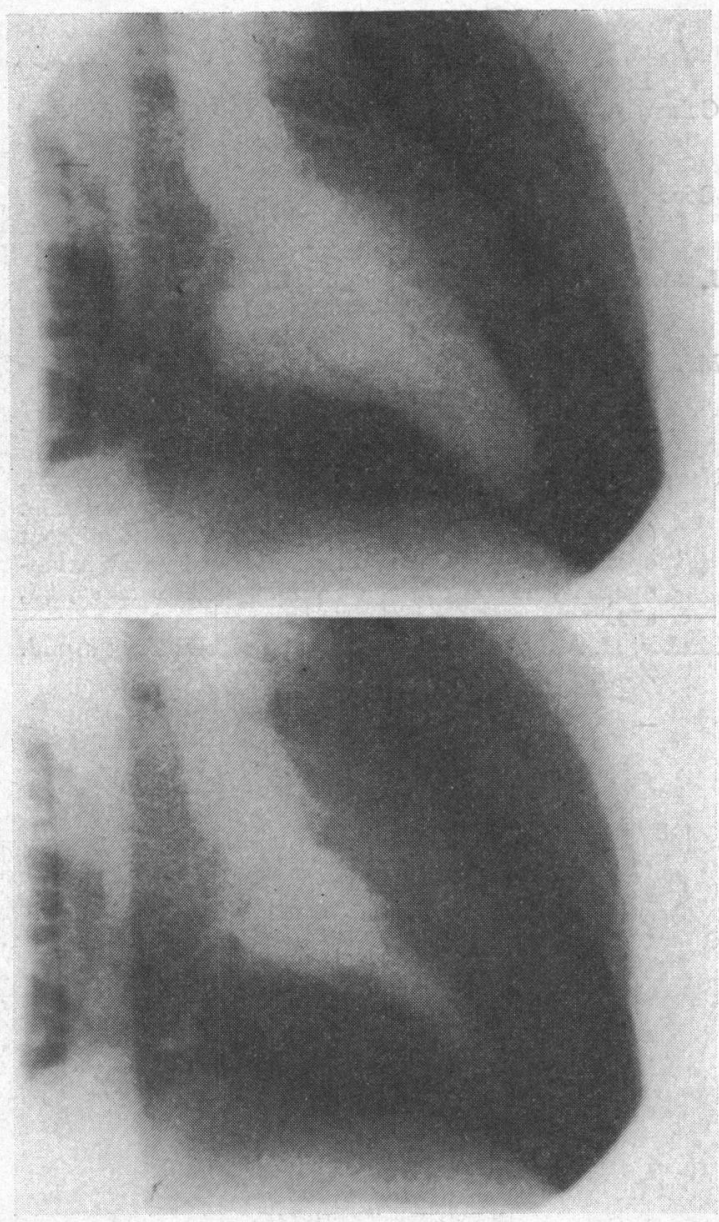

FIG. 7.-Right anterior oblique views in systole and diastole of the left ventricular cine-angiogram of Case 4. There is no narrowing of the left ventricular outflow tract. The ventricular cavity during systole appears constricted.

across the left ventricular outflow tract could not be shown at rest or during isoprenaline infusion, nor was outflow tract narrowing shown by cineangiography, but there was an abnormal configuration of the left ventricular cavity. The most severely affected members of this family belonged to older age-groups, and the authors suggested that the presence or absence of obstruction might depend on the duration of the disease. It appears possible that the patients we have described may also form a part of the spectrum of the same condition. It is not certain whether patients without obstruction will develop obstruction at a later stage of the disease, nor is the course of the disease known in the asymptomatic group, though sudden death has occurred in asymptomatic subjects. We feel that it is import- ant to recognize and follow up these marginal cases, for they may shed light upon the nature and prognosis of this interesting cardiomyopathy.

There were certain features present in these patients that merit special comment. Pulmonary hypertension was present in both of the twin sisters, and the mechanism appeared to be chronic left atrial hypertension secondary to a "stiff" left ventricle. Moderate pulmonary hypertension was present in 4 of the 24 patients described by Cohen et al. (1964) who had been studied by left and right heart catheterization, all 4 of whom had a left ventricular outflow tract systolic gradient.

Another interesting finding, which was present in 3 of our 4 patients, was the lack of movement of the components of the second heart sound with respiration. This finding was not reported in any of the patients suffering from hypertrophic obstructive cardiomyopathy who have been described. Boyer and Chisholm (1958), Shafter (1960), and Aygen and Braunwald (1962) have shown that, in normal subjects, inspiratory splitting of the second heart sound is caused by earlier aortic valve closure and later pulmonary valve closure due to a decrease of the left ventricular and an increase of the right ventricular stroke volume during inspiration. Shafter (1960) and Aygen and Braunwald (1962) have shown that, in atrial septal defect, there is little or no increase of the splitting in inspiration, because the two atria function as a common filling reservoir for both ventricles, and an increase in atrial inflow causes an increase of both the right and left ventricular stroke volume, and hence equal movement of both components of the second heart sound. The fixed splitting of the second heart sound in 3 of our 4 patients was similar to that seen in congestive heart failure, namely lack of movement of either component with inspiration (Leatham, Segal, and Shafter, 1963). It appears that reduced compliance of the ventricular wall is responsible for the lack of variation of the stroke volume of either ventricle with respiration, and hence for the fixed splitting of the second heart sound.

\section{SUMMARY}

Four patients with unexplained cardiac hypertrophy have been described. Two of the patients were identical twins, one had no apparent family history, and one had a family history of sudden death of many maternal relatives. In none of the patients was there ventricular outflow tract obstruction, though provocative tests were made only in one; but in all the left ventricular cavity had an abnormal configuration on angiography. It is suggested that such cases form a part of the wider spectrum of hypertrophic cardiomyopathy, some 
showing outflow tract obstruction, and some not showing this.

We are grateful to Dr. G. de J. Lee for permission to publish the reports of the first 3 cases, and to Dr. J. R. Hampton for information about the family history of Case 4. We also thank Professor J. F. Goodwin for reviewing the angiocardiograms.

\section{REFERENCES}

Aygen, M. M., and Braunwald, E. (1962). The splitting of the second heart sound in normal subjects and in patients with congenital heart disease. Circulation, 25, 328.

Boyer, S. H., and Chisholm, A. W. (1958). Physiologic splitting of the second heart sound. Circulation, 18, 1010.

Braunwald, E., Lambrew, C. T., Rockoff, S. D., Ross, J., Jr., and Morrow, A. G. (1964). Idiopathic hypertrophic subaortic stenosis. Circulation, 30, Suppl. IV, p. 3.

Burchell, H. B. (1964). Hypertrophic obstructive type of cardiomyopathy: Clinical syndrome. In Ciba Founda- tion Symposium: Cardiomyopathies, pp. 29-42. Ed. by G. E. W. Wolstenholme and M. O'Connor. Churchill, London.

Cohen, J., Effat, H., Goodwin, J. F., Oakley, C. M., and Steiner, R. E. (1964). Hypertrophic obstructive cardiomyopathy. Brit. Heart f., 26, 16.

Goodwin, J. F., Hollman, A., Cleland, W. P., and Teare, D. (1960). Obstructive cardiomyopathy simulating aortic stenosis. Brit. Heart f., 22, 403.

Leatham, A., Segal, B., and Shafter, H. (1963). Auscultatory and phonocardiographic findings in healthy children with systolic murmurs. Brit. Heart f., 25, 451.

McMichael, J. (1964). Chairman's Introduction. In Ciba Foundation Symposium: Cardiomyopathies, pp. 1-3. Ed. by G. E. W. Wolstenholme and M. O'Connor. Churchill, London.

Nasser, W. K., Williams, J. F., Mishkin, M. E., Childress, R. H., Helmen, C., Merritt, A. D., and Genovese, P. D. (1967). Familial myocardial disease with and without obstruction to left ventricular outflow: Clinical, hemodynamic, and angiographic findings. Circulation, 35, 638.

Shafter, H. A. (1960). Splitting of the second heart sound. Amer. F. Cardiol., 6, 1013. 